\title{
69. THE ORIGIN AND EVOLUTION OF THE COMETS AND OTHER SMALL BODIES IN THE SOLAR SYSTEM
}

\author{
S. K. VSEKHSVYATSKIJ \\ Astronomical Observatory, Kiev University, Kiev, U.S.S.R.
}

\begin{abstract}
It has become evident that comets and other small bodies are indications of eruptive evolution processes occurring in many of the planetary bodies of the solar system. The total number of near-parabolic comets moving in the solar system is $10^{11}$ to $10^{12}$, but as many as 10 to 15 percent of them are leaving the solar system with hyperbolic velocities. Taking into account also the number of short-period comets that degenerate into asteroids and meteor streams, we have estimated the total number of comets formed during the lifetime of the solar system as $10^{15}$ to $10^{16}$ (and total mass $10^{29}$ to $10^{31} \mathrm{~g}$ ). The investigation of comets and other small bodies enables us to evaluate the scale of the processes of cosmic vulcanism and the tremendous internal energy of the planets, that energy being derived from the initial stellar nature of planetary material.
\end{abstract}

\section{Introduction}

Most of the modern cosmogonical hypotheses consider the numerous minor bodies of the solar system to be of little importance in shedding light on the history of the system. These minor bodies are generally regarded as relics of the primeval matter from which the Sun and planets condensed (Kuiper, 1951) or as objects that condensed in the outer regions of the primordial nebula at distances of 3-50 AU and were then thrown out to the periphery of the solar system (Oort, 1963). The hypotheses do not explain the forces that ejected these objects into parabolic orbits and the manner in which the orbits later became circular - to produce the hypothetical Oort cloud of comets.

This particularly speculative idea, which is not based on any analysis of observational data, is refuted by everything known nowadays about the structure and physical nature of asteroids and meteorites. It also seems to me that the significance of collisions has been overrated; consider how much more important volcanic and tectonic processes are, not only on the Earth, but also on the Moon (NASA, 1969). These results all speak in favour of the fact that the minor bodies have formed as the result of volcanic processes in the planetary bodies.

\section{Data from Comets}

Examination of the cometary data gives more obvious information (Vsekhsvyatskij, $1962,1966,1967)$. The existence of planetary families of short-period comets that cannot be explained either by 'capture' or by 'diffusion', the nature of cometary gases, the extensive supplies of ice in cometary nuclei (where the meteorite fragments are), and several other facts also prove that the comets are the youngest objects in the solar system.

It is important to state some principal conclusions: 
(1) Analysis of the 'Laplace problem', statistics of cometary perihelia, and the kinematics of the cometary system leave no doubt that all comets, and consequently the products of their disintegration, were created within the solar system, and, on the average, more recently than the planets.

(2) The existence of the families of short-period comets of Jupiter, Saturn, Uranus, and Neptune, and in particular the fact that Jupiter's comets were invariably in the vicinity of Jupiter not too long before discovery, demonstrate the recent formation of these comets by eruption in the planetary system. Jupiter's satellites are likely to be the immediate source of the youngest comets of the Jupiter family. The recent detailed investigations by Everhart (1969) have quite definitely confirmed that it is impossible to explain the observed distribution of orbits of the short-period comets on the supposition of gravitational capture.

(3) The catalogues by Sekanina $(1966,1968)$ list 35 comets the future orbits of which are known to be hyperbolic, so that these objects must therefore leave the solar system. Several of the objects have high absolute brightness and masses up to $10^{19} \mathrm{~g}$. The amount of gaseous material alone lost by a comet of absolute magnitude $\mathrm{H}_{10}=0$ is $10^{12}$ to $10^{13} \mathrm{~g}$ per day, and the amount lost per revolution is typically $10^{15-0.4 \mathrm{H}_{10}}$ (Wurm, 1963). On the average one hyperbolic future orbit appears among every 4-6 nearly parabolic or long-period comets. This approximately corresponds to the results of van Woerkom (1948) on the accumulation of small perturbations. Taking into account the considerable number of comets with large perihelion distance $q$ and all the intrinsically faint comets, we find that the annual loss from the system of comets cannot be less than $10^{18} \mathrm{~g}$. During the whole period of existence of the solar system this corresponds to a loss of at least $10^{27} \mathrm{~g}$. Comparison with the estimated total number of comets $\left(10^{11}\right.$ to $\left.10^{12}\right)$ forces us to conclude that there must exist within the solar system sources for replenishing the cometary objects. Considering the number of comets that have left the solar system and the number of disintegrated short-period comets (which turned into asteroids, meteorites, and meteoroids), we estimate the total number of comets (with mean mass $10^{14}$ to $10^{15} \mathrm{~g}$ ) created since the origin of the solar system as $10^{15}$ to $10^{16}$. The total mass of material ejected from the surfaces of the planets is therefore $10^{29}$ to $10^{31} \mathrm{~g}$.

(4) The existence of comets and other minor bodies provides the possibility of estimating the extent of cosmic vulcanism processes in the history of the solar system and the internal energy of the planets (some $10^{41}$ to $10^{43} \mathrm{erg}$ ) expended in the ejection of considerable quantities of planetary material into interplanetary and interstellar space.

These conclusions, made long before the space age began, led investigators to anticipate the high volcanic activity on the surfaces of the planets. Volcanic activity, not only on the giant planets, but also on Venus and Mars, has attracted attention, and the missions to the last-mentioned planets and the Moon have demonstrated the decisive role of volcanic processes in the evolution of planetary bodies. At the same time, recent investigations clearly confirm the existence of meteoritic masses in cometary nuclei and consequently that comets are fragments of the crusts and the frozen atmospheres of the planets. 


\section{Planetary Densities}

It is clear that of all the physical characteristics of the planets, the one of greatest significance for studying the problem of the comets and other minor bodies is mean density. The physical and dynamical evolution of the planetary bodies took place as the result of loss of the lighter elements from the surface layers. This resulted in an increase in mean density. The absence nowadays of stellar abundances of hydrogen and helium in the terrestrial planets suggests that the amount of mass lost greatly exceeds the modern masses of these planets.

We may therefore use the data on mean density to establish the scale of the creation processes of comets, meteorites, and asteroids. The masses $M$, mean densities $\bar{\rho}_{c}$, and specific rotational energies $E_{R}=\frac{1}{2} I \omega^{2} / M$ (where $I$ is the moment of inertia about the axis of rotation and $\omega$ is the angular velocity of rotation) for various bodies are listed in Table I (Vsekhsvyatskij, 1971). It is apparent that there are two analogous

TABLE I

Masses, densities, and specific rotational energies

\begin{tabular}{lccl}
\hline & $M(\mathrm{grams})$ & $\bar{\rho}_{c}\left(\mathrm{~g} \mathrm{~cm}^{-3}\right)$ & $E_{R}\left(\mathrm{erg} \mathrm{g}^{-1}\right)$ \\
\hline Sun & $2.0 \times 10^{33}$ & 1.41 & $9.6 \times 10^{8}$ \\
Jupiter & $1.9 \times 10^{30}$ & 1.39 & $2.6 \times 10^{11}$ \\
Saturn & $5.7 \times 10^{29}$ & 0.71 & $1.7 \times 10^{11}$ \\
Uranus & $8.7 \times 10^{28}$ & 1.6 & $2.5 \times 10^{10}$ \\
Neptune & $6.1 \times 10^{28}$ & 1.6 & $1.0 \times 10^{10}$ \\
Earth & $6.0 \times 10^{27}$ & 5.51 & $3.7 \times 10^{8}$ \\
Pluto & $5.5 \times 10^{27}$ & 10 & $9.5 \times 10^{8}$ \\
Venus & $4.9 \times 10^{27}$ & 5.3 & $5.1 \times 10^{3}$ \\
Mars & $6.4 \times 10^{26}$ & 4.0 & $9.4 \times 10^{7}$ \\
Mercury & $3.3 \times 10^{26}$ & 5.8 & $2.1 \times 10^{4}$ \\
Ganymede & $1.6 \times 10^{26}$ & 2.4 & $2.4 \times 10^{6 \mathrm{a}}$ \\
Titan & $1.4 \times 10^{26}$ & 2.3 & $2.4 \times 10^{6 \mathrm{a}}$ \\
Triton & $8.7 \times 10^{25}$ & 2.0 & $1.6 \times 10^{6 \mathrm{a}}$ \\
Moon & $7.4 \times 10^{25}$ & 3.35 & $3.6 \times 10^{3}$ \\
Callisto & $9.7 \times 10^{25}$ & 2.1 & $10^{6}-10^{5 \mathrm{a}}$ \\
Io & $7.2 \times 10^{25}$ & 4.0 & $10^{6}-10^{5 \mathrm{a}}$ \\
Europa & $4.7 \times 10^{25}$ & 3.8 & $10^{6}-10^{5 \mathrm{a}}$ \\
\hline
\end{tabular}

a The period of revolution is adopted.

groups, the terrestrial planets and the Galilean satellites, each showing a decrease in mean density with increasing distance from the central body. This is regarded as an important indication of the eruptive evolution of the planets, proceeding most rapidly under the tidal influence of the central bodies.

The distribution of density in a planet is given by

$$
\rho=\rho_{0}\left(1-\xi r^{\lambda}\right)
$$

where $\rho_{0}$ is the central density, $r$ is the fraction of the radius, varying from 0 at the 
centre to 1 at the surface, and $\xi$ and $\lambda$ are parameters. From this we may obtain the present mean density,

$$
\bar{\rho}_{c}=\rho_{0}\left(1-\frac{3 \xi}{\lambda+3} r^{\lambda}\right)
$$

which enables us to estimate the initial radius $r$ and establish the initial dimensions of the planet.

For the initial mean density of protoplanets we may take the present mean density of Jupiter or of the Sun, because their relative mass loss cannot be very significant. For the Earth we take $\rho_{0}=12 \mathrm{~g} \mathrm{~cm}^{-3}$, and it then follows that the initial radius was in the range 14 to 7.1 thousand kilometres, the loss of mass being anywhere between 2 to 3 and 0.2 times the present mass of the Earth. The loss of mass from Venus would be of the same order; much more mass could have been lost from Pluto - if the high mean density for this planet is correct.

The total amount of material ejected from all the planets since the origin of the solar system could exceed $10^{29}$ to $10^{30} \mathrm{~g}$. This value is of the same order as the total mass of comets and other minor bodies created during the history of the solar system. This shows that inside the planets there must have been powerful energy sources far greater than anything derived from gravitational collapse or radioactive decay. It is quite natural to suppose that the energy supply was preserved from the initial stellar condition of the planetary material.

\section{Minor Planets}

The data from the system of asteroids agree well with our conclusions from the system of comets. Table II shows the distribution of the absolute magnitudes $g$ of the 1746 permanently numbered objects. The first seven values, up to and including that for $g=10-11$, probably characterize the real distribution of asteroidal sizes in the accessible

TABLE II

Distribution of absolute magnitudes of minor planets

\begin{tabular}{lllllllll}
\hline$g$ & $4-5$ & $5-6$ & $6-7$ & $7-8$ & $8-9$ & $9-10$ & $10-11$ & $11-12$ \\
\hline 2 & 1 & 6 & 23 & 87 & 209 & 345 & 395 \\
\hline$g$ & $12-13$ & $13-14$ & $14-15$ & $15-16$ & $16-17$ & $17-18$ & $18-19$ & $19-20$ \\
\hline 332 & 230 & 95 & 13 & 6 & 1 & 0 & 1 \\
\hline
\end{tabular}

region of space (up to $5.5 \mathrm{AU}$ from the Sun), and from them we may obtain the following dependence of the number $n$ and the mass $M$ :

$$
n=0.000651 \times 2.94^{g}
$$

$\log M=26.74-0.6 g$. 
Figure 1 also shows the distribution, the above expression for $n$ being shown by the broken line.

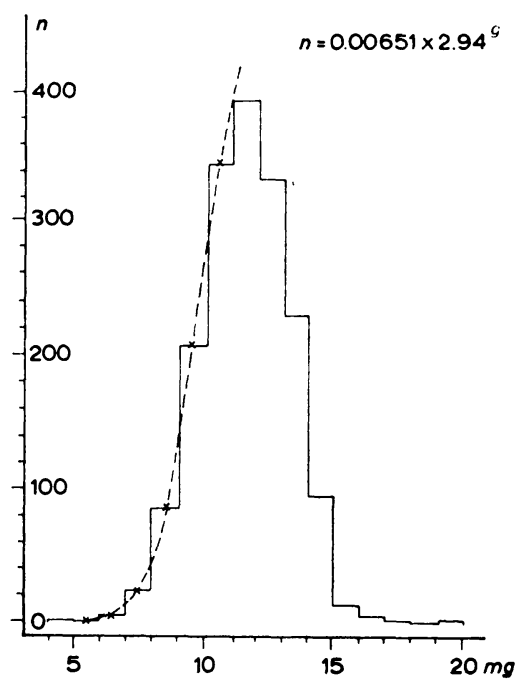

Fig. 1. Distribution of absolute magnitudes $g$ of the minor planets. The broken line shows the distribution extrapolated from the brighter objects.

The extrapolated number of minor planets of $g=19.5$ is thus $n=7.6 \times 10^{6}$, and their combined mass is $M=10^{19}-10^{20} \mathrm{~g}$, from which it follows that the total mass of the asteroids up to $g=19-20$ is of the order $10^{26}$ to $10^{27} \mathrm{~g}$. Considering that the total volume of the planetary system and the volume of the visibility region are in the ratio $(40 / 5)^{3} \simeq 500$, we deduce that the total mass of asteroidal material in the solar system is some $10^{28}$ to $10^{29} \mathrm{~g}$, which is again approximately consistent with the cometary data.

\section{Evidence from Meteoroids}

According to Brandt and Hodge (1964) some $10^{7}$ to $10^{9} \mathrm{~g}$ of meteoroidal matter encounter the Earth daily and are pulverized in its atmosphere. Taking into account the Earth's attraction, this corresponds to a volume of approximately $10^{15} \mathrm{~km}^{3}$. Supposing meteoroid density to be the same over the volume occupied by the system of planets $\left(10^{30} \mathrm{~km}^{3}\right)$ the total mass of meteoroids must amount to $10^{23} \mathrm{~g}$. Since these particles move along the cometary orbits, orbiting the Sun in decades or at most a few centuries, we may deduce that the total amount of finely dispersed substance produced during the existence of the solar system cannot be less than $10^{23} \times 10^{6}$ to $7=$ $10^{29}$ to $10^{30} \mathrm{~g}$.

Among photographic and radio meteors a significant proportion have small perihelion distances $(q<0.4 \mathrm{AU})$ and large eccentricities; the majority have aphelion distances not exceeding 3 to $4 \mathrm{AU}$ (Vsekhsvyatskij, 1967). More than a quarter of all 
the particles move in rather eccentric orbits relatively near the Sun. The PoyntingRobertson effect implies that the lifetimes of such particles are rather small and measured only in hundreds or at most thousands of years. Solar corpuscular radiation is still more effective at dispersing and sweeping out these particles. We conclude that the particles observed near the Sun arise in the inner region of the solar system. It seems impossible to explain their character by capture from long-period orbits or their creation as a result of the disintegration of comets.

It has already been suggested that objects with orbits of small perihelion distance and small semimajor axis (e.g., P/Encke, P/Wilson-Harrington, Icarus, the Apollo asteroids, etc.) could arise as a result of eruptive processes on Venus, the space missions having indicated temperatures there of the order $800 \mathrm{~K}$, pressures of more than $100 \mathrm{~atm}$, a large amount of dust in the upper atmosphere, and rapidly varying dark features above the cloud cover that appear to consist of clouds of volcanic ash.

The peculiarities of all the groups of minor bodies thus illustrate the rapid dynamical and physical evolution especially evident in the case of comets and meteoroids. Together with the results of analysis of meteorites they give evidence of the processes of ejection from the surfaces of satellites and planets (the Moon, the satellites of Jupiter and Saturn, Venus, Mars, and the Earth).

\section{References}

Brandt, J. and Hodge, P.: 1964, Solar System Astrophysics, New York and London.

Everhart, E.: 1969, Astron. J. 74, 735.

Kuiper, G. P.: 1951, in J. A. Hynek (ed.), Astrophysics, McGraw-Hill, New York, Toronto and London, p. 400.

NASA: 1969, Apollo 11 : Preliminary Science Report.

Oort, J. H.: 1963, in The Moon, Meteorites and Comets, Vol. IV of the series: The Solar System (ed. by B. M. Middlehurst and G. P. Kuiper), University of Chicago Press, Chicago and London, p. 665 .

Sekanina, Z.: 1966, Publ. Astron. Inst. Charles Univ. No. 48.

Sekanina, Z.: 1968, Publ. Astron. Inst. Charles Univ. No. 56.

van Woerkom, A. J.: 1948, Bull. Astron. Inst. Neth. 10, 445.

Vsekhsvyatskij, S. K.: 1962, Publ. Astron. Soc. Pacific 74, 106.

Vsekhsvyatskij, S. K.: 1966, Mem. Soc. Roy. Sci. Liège Sez. 5 12, 469.

Vsekhsvyatskij, S. K.: 1967, Priroda i Proiskhozhdenie Komet i Meteornogo Veshchestva, Prosveshchenie, Moscow.

Vsekhsvyatskij, S. K.: 1971, Probl. Kosmich. Fiz. No. 6.

Wurm, K.: 1963, in The Moon, Meteorites and Comets, Vol. IV of the series: The Solar System (ed. by B. M. Middlehurst and G. P. Kuiper), University of Chicago Press, Chicago and London, p. 573 . 